\title{
Noncoding Transcripts in Sense and Antisense Orientation Regulate the Epigenetic State of Ribosomal RNA Genes
}

\author{
H. Bierhoff, K. Schmitz, F. MaAss, J. Ye, AND I. Grummt \\ Division of Molecular Biology of the Cell II, German Cancer Research Center, DKFZ-ZMBH Alliance, \\ D-69120 Heidelberg, Germany \\ Correspondence: i.grummt@dkfz.de
}

\begin{abstract}
Alternative transcription of the same gene in sense and antisense orientation regulates expression of protein-coding genes. Here we show that noncoding RNA (ncRNA) in sense and antisense orientation also controls transcription of rRNA genes (rDNA). rDNA exists in two types of chromatin - a euchromatic conformation that is permissive to transcription and a heterochromatic conformation that is transcriptionally silent. Silencing of rDNA is mediated by NoRC, a chromatin-remodeling complex that triggers heterochromatin formation. NoRC function requires RNA that is complementary to the rDNA promoter (pRNA). pRNA forms a DNA:RNA triplex with a regulatory element in the rDNA promoter, and this triplex structure is recognized by DNMT3b. The results imply that triplex-mediated targeting of DNMT3b to specific sequences may be a common pathway in epigenetic regulation. We also show that rDNA is transcribed in antisense orientation. The level of antisense RNA (asRNA) is down-regulated in cancer cells and up-regulated in senescent cells. Ectopic asRNA triggers trimethylation of histone $\mathrm{H} 4$ at lysine 20 (H4K20me3), suggesting that antisense transcripts guide the histone methyltransferase Suv4-20 to rDNA. The results reveal that noncoding RNAs in sense and antisense orientation are important determinants of the epigenetic state of rDNA.
\end{abstract}

The past few years have witnessed the importance of epigenetics in the control of gene expression. A wealth of recent evidence points to the indispensable role of noncoding RNAs (ncRNAs) in chromatin-based processes, demonstrating that epigenetic regulation represents an intimate and balanced interplay of both RNA and chromatin fields (Mattick 2009; Malecová and Morris 2010). Noncoding RNAs are numerous and highly adapted in processes that require specific nucleic acid recognition without complex catalysis, such as in guiding and modifying nucleic acids or in directing posttranscriptional regulation of gene expression and chromatin structure. Although the mechanistic details as to how RNA and chromatin are connected remain unclear, several long ncRNAs have been implicated in transcriptional silencing and changing chromatin structure either at single loci, across large chromosomal regions, or from entire chromosomes (Avner and Heard 2001; Sleutels et al. 2002; Nagano and Fraser 2009). Thus, the primary role of ncRNA in epigenetic regulation might be to recruit chromatin-modifying enzymes to restrict DNA methylation and specific histone modifications to particular regions of the genome.

Previous studies have established that rRNA genes exist in two epigenetic conformations, transcriptionally active genes exhibiting euchromatic features, whereas transcriptionally silent ones are hypermethylated and marked by heterochromatic histone modifications (Santoro et al. 2002). The fact that even in proliferating cells with a high demand for ribosome biogenesis a significant fraction of rRNA genes is epigenetically silent, provides a unique possibility to decipher the mechanisms that establish the euchromatic and heterochromatic state of rRNA genes and to study the consequence of balancing the ratio of epigenetically active and silent rDNA repeats for cell surveillance and genomic stability. The silent, heterochromatic state of rDNA is accomplished by the nucleolar remodeling complex NoRC, an ATP-dependent chromatin-remodeling complex comprising the ATPase SNF2h and a large subunit, termed TIP5 (TTF-I-interacting protein \#5) (Strohner et al. 2001). NoRC silences a subset of rRNA genes by recruiting chromatin-modifying enzymes to the rDNA promoter that are required for heterochromatin formation and transcriptional silencing (Zhou et al. 2002; Zhou and Grummt 2005). Moreover, NoRC triggers de novo DNA methylation of the rDNA promoter. Methylation impairs binding of the RNA polymerase I (Pol I)-specific transcription factor UBF, a process that nucleates the assembly of transcription initiation complexes (Santoro and Grummt 2001). Thus, NoRC coordinates the activities of several macromolecular complexes that modify histones, methylate DNA, and establish a "closed" heterochromatic state that is refractory to transcription. Significantly, NoRC is associated with RNA that originates from a Pol I promoter in the intergenic spacer, and this RNA, termed "pRNA," is indispensable for NoRC function (Mayer et al. 2006). In addition, a fraction of rDNA is transcribed in antisense orientation, and these antisense transcripts promote trimethylation of histone $\mathrm{H} 4$ at lysine 20 (H4K20me3). In this chapter, we discuss our knowledge on the role of noncoding RNA in epigenetic control of rRNA genes, deciphering mechanisms that may be common and widely used in epigenetic regulation. 


\section{INTERGENIC TRANSCRIPTS ARE REQUIRED FOR NoRC-DEPENDENT SILENCING OF RRNA GENES}

The tandemly repeated rRNA genes are separated by long intergenic spacers containing essential sequence elements that are required for transcription initiation, termination, transcriptional enhancement, and recombination. Studies in several organisms have shown that the intergenic spacer contains duplicated sequences that resemble the core element of the major gene promoter and direct Pol I transcription in vitro and in vivo (Moss et al. 1980; Cassidy et al. 1987; Kuhn and Grummt 1987). The function of the spacer promoter and the role of intergenic transcripts are poorly understood. In mouse, transcripts from the spacer promoter located $\sim 2 \mathrm{~kb}$ upstream of the pre-rRNA start site are $\sim 1000$-fold less abundant than pre-rRNA and are hard to detect in vivo (Santoro et al. 2010). Nevertheless, these intergenic transcripts have an important role in establishing the silent state of rRNA genes. The primary intergenic transcripts are degraded by the exosome, however, a heterogeneous population of 150-250-nucleotide RNA that is complementary to the rDNA promoter, termed "pRNA," is shielded from degradation by binding to the chromatinremodeling complex NoRC. NoRC consists of two subunits, the ATPase SNF2h and TIP5, a $>200-\mathrm{kDa}$ protein that shares sequence similarity to large subunits of other SNF2h-containing chromatin-remodeling complexes, e.g., RSF, ACF, or WSTF (Strohner et al. 2001). pRNA associates with NoRC by interaction with the TAM (TIP5/ ARBD/MBD) domain of TIP5, which exhibits sequence homology to the methyl-CpG-binding domain (MBD), present in proteins that bind to methylated DNA, and can recruit corepressors that generate heterochromatic features (Hendrich and Tweedie 2003; Bogdanovic and Veenstra 2009). NoRC silences rRNA genes by targeting chromatinmodifying enzymes to the rDNA promoter that alter the chromatin structure and impair transcription complex assembly (Santoro and Grummt 2001; Santoro et al. 2002; Zhou et al. 2002; Zhou and Grummt 2005). RNase treatment or knockdown of pRNA abrogates NoRC occupancy at rDNA and impairs methylation of the rDNA promoter and heterochromatin formation, underscoring the functional relevance of pRNA in NoRC-dependent rDNA silencing. Thus, pRNA can be added to the growing list of functional ncRNAs that originate from processed introns or intergenic regions and have distinct roles in the regulation of gene expression.

NoRC-associated pRNA is synthesized from a subset of rDNA repeats during mid $\mathrm{S}$ phase, acting in trans to propagate DNA methylation and transcriptional repression of late-replicating silent rDNA copies (Santoro et al. 2010). NoRC recognizes a phylogenetically conserved secondary structure of pRNA (Mayer et al. 2008). Mutations that disrupt a specific stem-loop of pRNA abrogate the interaction with TIP5 and abolish targeting of NoRC to nucleoli. Consistently, compensatory mutations that allow formation of the hairpin structure restore binding to TIP5 and nucleolar localization of NoRC. A TIP5 mutant that contains two amino acid exchanges in the TAM domain is deficient in RNA-binding, indicating that the integrity of the TAM do- main is required for the association of NoRC with pRNA (Mayer et al. 2006). Strikingly, none of the typical heterochromatic histone modifications were induced by mutant TIP5, indicating that the establishment of heterochromatic features at the rDNA promoter requires the association of NoRC with pRNA. Surprisingly, this RNA-binding-deficient mutant was still capable of triggering de novo $\mathrm{CpG}$ methylation and transcriptional silencing, indicating that DNA methylation rather than repressive chromatin modifications causes silencing. This is consistent with previous results showing that mutation of a single $\mathrm{CpG}$ residue in the rDNA promoter alleviates NoRC-mediated transcriptional repression without affecting heterochromatin formation, indicating that heterochromatin formation can be uncoupled from DNA methylation.

The amount of cellular pRNA is proportional to the amount of TIP5, indicating that pRNA is stabilized by binding to NoRC. Overexpression of TIP5 resulted in decreased levels of $45 \mathrm{~S}$ pre-rRNA and elevated amounts of pRNA, whereas RNAi-mediated depletion of TIP5 increased prerRNA synthesis and reduced the level of pRNA (Mayer et al. 2006). These results are reminiscent of dosage compensation in Drosophila male flies, where roX RNAs that recruit MSL proteins are highly unstable unless the MSL complex is coexpressed (Meller et al. 2000). Knockdown of pRNA by antisense locked nucleic acid (LNA)/DNA gapmers released NoRC from nucleolar chromatin and increased pre-rRNA synthesis, consistent with pRNA targeting NoRC to chromatin and repressing rDNA transcription (Fig. 1A,B). The increase in pre-rRNA levels correlates with decreased $\mathrm{CpG}$ methylation, increased euchromatic histone marks (such as $\mathrm{H} 3 \mathrm{~K} 4 \mathrm{me} 3$ ), and reduced heterochromatic histone modifications (such as H4K20me3) at the rDNA promoter (Fig. 1C). Conversely, elevation of pRNA levels led to de novo DNA methylation, heterochromatin formation, and transcriptional silencing. The intriguing correlation between the amount of NoRC and pRNA, on the one hand, and the inverse correlation between pRNA and rDNA transcription, on the other hand, emphasizes the relevance of pRNA in NoRC-dependent epigenetic control of rDNA repeats.

\section{ACETYLATION REGULATES INTERACTION OF TIP5 WITH PRNA}

Studies in Saccharomyces cerevisiae and Drosophila melanogaster have demonstrated that rDNA silencing has an important role in genome stability through the suppression of nonhomologous recombination. Loss of silencing correlates with rDNA instability, nucleolar disintegration, and cellular senescence (Kobayashi et al. 2004; Peng and Karpen 2007). Because NoRC ensures rDNA stability by mediating heterochromatin formation and gene silencing, it is conceivable that NoRC activity is modulated in response to external signals that affect cell metabolism. Indeed, TIP5 is acetylated by the acetyltransferase MOF (males absent on the first) at a single lysine residue (K633) adjacent to its RNA-binding domain, and acetylation is required for NoRC-dependent rDNA silencing (Zhou et al. 2009). Mutation of K633 rendered TIP5 in- 
A

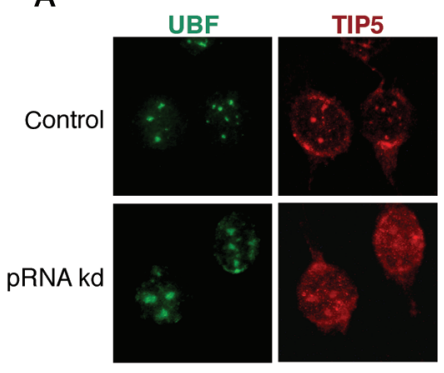

B

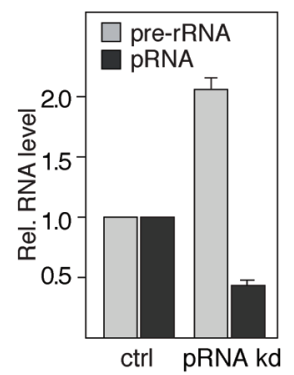

C

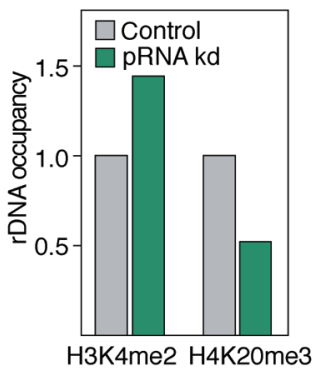

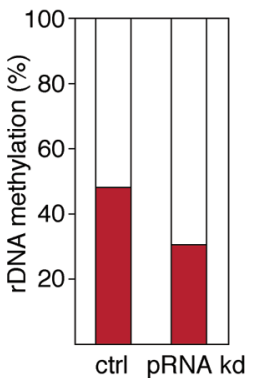

Figure 1. Intergenic transcripts are required for targeting NoRC to nucleoli, rDNA silencing, de novo DNA methylation, and heterochromatin formation. $(A)$ Nucleolar localization of NoRC requires pRNA. NIH3T3 cells were depleted from pRNA by antisense LNADNA gapmers comprising mouse rDNA sequences from -99 to -81 (pRNA kd), and localization of TIP5 and UBF was monitored by indirect immunofluorescence. As a control, cells were transfected with unspecific LNA-DNA gapmers. (B) Silencing of rDNA depends on pRNA. Reverse transcriptase-quantitative polymerase chain reaction (RT-qPCR) data showing the level of pre-rRNA (light bars) and pRNA (dark bars) in cells treated with control or pRNA-specific antisense LNA-DNA gapmers. (C) pRNA mediates heterochromatin formation and rDNA methylation. (Left) Chromatin immunoprecipitation (ChIP) data showing H3K4me3 and H4K20me3 occupancy at the rDNA promoter in cells transfected with control (gray bars) or pRNA-specific antisense LNA-DNA gapmers (green bars). (Right) Genomic DNA from control or pRNA knockdown cells was digested with HpaII and subjected to qPCR using primers that flank an HpaII site at -143 in the rDNA promoter. Diagram shows ratio of methylated (red) to HpaII-sensitive, unmethylated rRNA (white) genes.

capable of shifting the position of the promoter-bound nucleosome and, additionally, mediating heterochromatic histone modifications, triggering $\mathrm{CpG}$ methylation, and repressing transcription. Unexpectedly, the acetylation-deficient mutant bound to pRNA with higher affinity than wild-type TIP5. Consistently, acetylation by MOF strongly reduced the pRNA-binding activity of TIP5, indicating that reversible acetylation regulates the association of pRNA with NoRC. Searching for enzymes that counteract MOF-dependent acetylation of TIP5, we found that the $\mathrm{NAD}^{+}$-dependent deacetylase SIRT1 specifically removes the acetyl group from K633, allowing the association of pRNA with NoRC. Thus, acetylation by MOF and deacetylation by SIRT1 govern the binding of pRNA to TIP5, a process that is instrumental for NoRC-dependent rDNA silencing. In support of this view, elevation of the cellular $\mathrm{NAD}^{+} / \mathrm{NADH}$ ratio following glucose deprivation led to activation of SIRT1, deacetylation of TIP5, increase in pRNA levels, and repression of rDNA transcription. These results reinforce the functional link between TIP5 acetylation, pRNA binding, and rDNA silencing and highlight the close interrelationship among the acetyltransferase $\mathrm{MOF}$, the $\mathrm{NAD}^{+}$-dependent deacetylase SIRT1, and the chromatin-remodeling complex NoRC (Fig. 2). Of note, the involvement of SIRT1 in the silencing process provides an intriguing opportunity to link the cellular energy status to rDNA silencing, a process that maintains nucleolar integrity and ensures cell survival.

\section{PRNA RECRUITS DNMT3B TO RDNA}

Analysis of the silencing capacity of wild-type and mutant forms of pRNA revealed that sequences comprising the stem-loop structure that is recognized by NoRC are essential for triggering heterochromatic histone modifications but are not required for DNA methylation and transcriptional silencing (Fig. 3A). Silencing requires upstream pRNA sequences that are complementary to the promoterproximal terminator $\mathrm{T}_{0}$. Truncated $\mathrm{pRNA}$ derivatives lacking the $\mathrm{T}_{0}$ sequence fail to trigger nucleolar localization of

\begin{tabular}{|c|c|c|c|c|c|c|}
\hline $531 / 532$ & 633 & & & & & 75 \\
\hline \multirow{3}{*}{$\begin{array}{l}\text { rDNA association } \\
\text { Nucleosome } \\
\text { positioning }\end{array}$} & $\begin{array}{c}\text { Wild } \\
\text { type }\end{array}$ & $\begin{array}{c}\text { WY } \\
531 / 532\end{array}$ & K633 & $\begin{array}{l}\text { WYK } \\
633\end{array}$ & Y1775 & \\
\hline & + & $-/+$ & + & nd & - & \\
\hline & + & + & - & + & - & \\
\hline RNA binding & + & - & + & - & + & T \\
\hline $\begin{array}{l}\text { Heterochromatic } \\
\text { marks }\end{array}$ & + & - & $-1+$ & nd & - & Bromo \\
\hline DNA methylation & + & + & - & + & - & \\
\hline Tx silencing & + & + & - & + & - & \\
\hline
\end{tabular}

Figure 2. NoRC-dependent silencing of rDNA. Modular organization and location of sequence motifs in TIP5 that have been associated with functions in NoRC-mediated rDNA silencing. Carboxy-terminal part of TIP5 contains a tandem PHD finger and bromodomain that binds to H4K16ac and interacts with histone deacetylases (HDACs) and histone methyltransferases (HMTs). TTF-I associates with the part of TIP5 comprising amino acids 598-731. Four AT hooks mediate nonspecific DNA binding. pRNA binds to TIP5 by interaction with the TAM domain. Table summarizes the ability of TIP 5 mutants to bind to rDNA, remodel nucleosomes, associate with pRNA, and promote heterochromatin formation, DNA methylation, and transcriptional repression (Zhou and Grummt 2005; Mayer et al. 2006; Zhou et al. 2009). Numbers refer to positions of amino acids that have been mutated. 

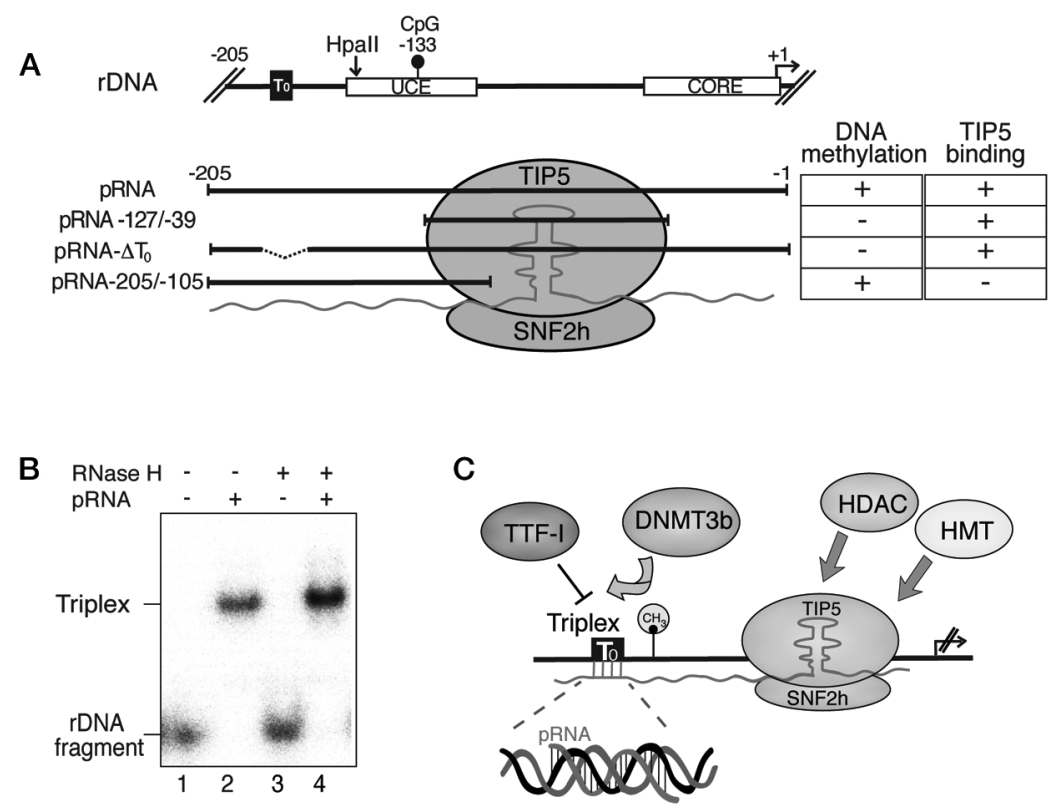

Figure 3. pRNA sequences upstream of the NoRC-binding site are required to mediate hypermethylation of the rDNA promoter. $(A)$ rDNA promoter and pRNA mutants used. Marked are the promoter-proximal terminator $\mathrm{T}_{0}$, upstream control element (UCE), and core promoter. Numbers refer to positions of nucleotides with respect to transcription start site. Highlighted is the pRNA sequence forming the stem-loop structure (nucleotides from -127 to -39) that is recognized by NoRC. (Right) Ability of pRNA mutants to associate with TIP5 and to mediate methylation of the rDNA promoter. $(B)$ pRNA forms a DNA:RNA triplex structure with rDNA. Synthetic pRNA was incubated with a ${ }^{32} \mathrm{P}-$-labeled rDNA promoter fragment $(-205 /-140)$, and formation of DNA:RNA triplexes was monitored by an electrophoretic mobility shift assay (EMSA) (lanes 1,2). To exclude RNA:DNA hybrid formation, preformed rDNA:pRNA triplexes were treated with 30 units of RNase $\mathrm{H}(\mathrm{H})$ (lanes 3, 4). (C) Model of triplex-mediated rDNA methylation and transcriptional silencing. pRNA (red) forms a triplex structure with $\mathrm{T}_{0}$, thereby displacing transcription factor TTF-I from its binding site. Triplex structure targets DNMT3b to the rDNA promoter, leading to methylation of CpG-133 and inhibition of transcription complex assembly. Interaction of TIP5 with HDACs and HMTs generates a heterochromatic structure that consolidates rDNA repression.

NoRC and rDNA silencing (Schmitz et al. 2010). However, a pRNA version lacking the stem-loop structure that is required for the interaction with TIP5 (nucleotides from -127 to -39) was as efficient as full-length pRNA in inducing hypermethylation of rDNA and transcriptional silencing. This indicates that pRNA itself is capable of mediating methylation of the rDNA promoter, provided that it contains sequences matching $\mathrm{T}_{0}$. In vitro and in vivo experiments revealed that a stretch of 20 nucleotides in pRNA is able to form a triple-stranded structure with the $T_{0}$ sequence via Hoogsteen or reversed Hoogsteen base-pairing (Fig. 3B). This DNA:RNA triplex is specifically recognized by the de novo DNA methyltransferase DNMT3b. The finding that DNMT3b exhibits binding specificity for DNA:RNA triplexes suggests a compelling new mechanism of epigenetic regulation that involves DNA:RNA triplexes in targeting DNA methyltransferase to regulatory gene sequences (Fig. 3C).

Triple-helix target sites, mostly stretches of oligopyrimidine/oligopurine sequences in the DNA target, are overrepresented in the human genome, especially at promoter regions, highlighting the potential of naturally occurring hydrogen-bond interactions of ncRNA with the major groove of DNA in the control of gene expression (Goñi et al. 2004; Belotserkovskii et al. 2007; Martianov et al. 2007). Triplex formation has been shown to contribute to promoter-specific transcriptional repression by compro- mising transcription factor binding (Kim and Miller 1998; Martianov et al. 2007). Likewise, formation of a DNA: RNA triplex structure at $\mathrm{T}_{0}$ leads to displacement of TTF$\mathrm{I}$, the factor that binds to $\mathrm{T}_{0}$ and participates in NoRC recruitment (Evers et al. 1995; Strohner et al. 2004). Dissociation of TTF-I, in turn, permits the interaction of DNMT3b with the triplex structure, raising the exciting possibility that triplex-dependent recruitment of DNMT3b to specific gene sequences might be a common function of regulatory ncRNAs. A triplex-mediated mechanism of DNA methylation and gene silencing is particularly intriguing in view of the finding that $>90 \%$ of the transcriptional output of the human genome represents ncRNA and many ncRNAs function in trans to induce epigenetic changes (Khalil et al. 2009). Thus, ncRNAs may have an important role in regulating gene expression by forming DNA:RNA triplex structures, thereby guiding DNMTs and other silencing factors to their respective target sequences.

\section{RDNA IS TRANSCRIBED IN ANTISENSE ORIENTATION BY RNA POL II}

Recent transcriptome analysis has revealed that expression of mammalian genomes comprises a large extent antisense transcription, with up to $72 \%$ of sense transcripts having antisense partners. In several cases, antisense RNAs 
A

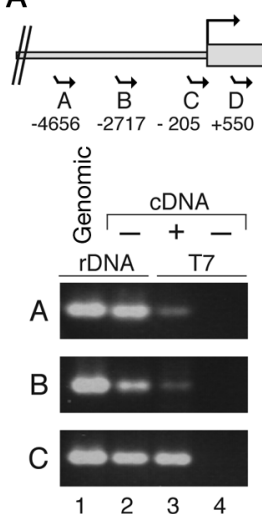

B

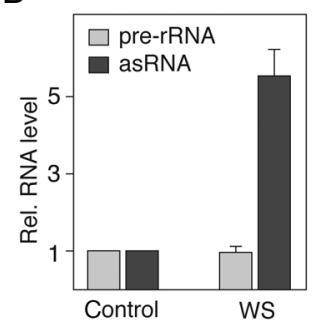

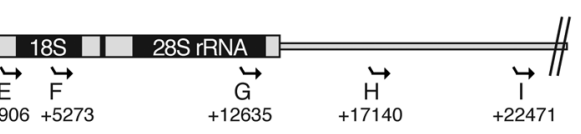
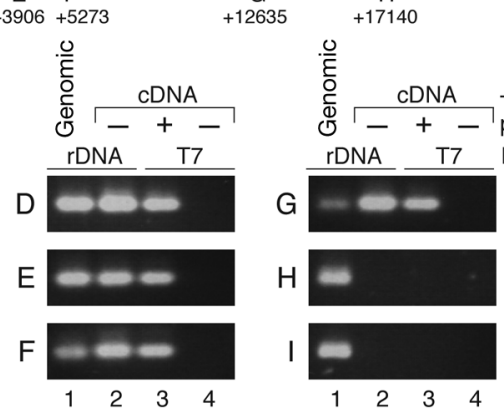

C

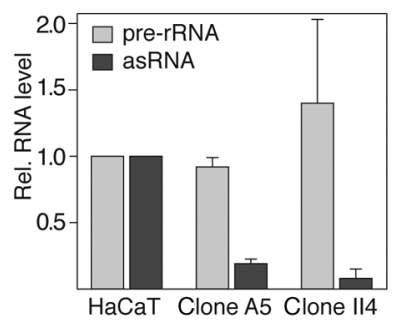

Figure 4. rDNA is transcribed in antisense orientation. (A) Detection of antisense transcripts by RT-PCR. RNA from NIH3T3 cells was reverse-transcribed using either no primer (lanes 2, 4) or rDNA-specific forward primers (A-I) linked to the T7 promoter (lane 3). For PCR amplification, an rDNA reverse primer was used together with either an rDNA-specific forward primer (lanes 1,2) or a T7 primer (lanes 3, 4). Genomic DNA from NIH3T3 cells served as a control (lane 1). PCR products were resolved on agarose gels and visualized by ethidium bromide staining. (Above) rDNA repeat and position of primers A-I used. (B) Antisense transcripts are up-regulated in fibroblasts from Werner syndrome (WS) patients. RNA from control or WS fibroblasts was reverse-transcribed, and asRNA and pre-rRNA were determined by real-time PCR using SYBR Green. Values for WS fibroblasts were normalized to control human fibroblasts. (C) Levels of asRNA and pre-rRNA in a progressive skin carcinogenesis model. asRNA and pre-rRNA were monitored in nontumorigenic HaCaT keratinocytes and in transformed HaCaT derivatives, clones A5 and II4. Clone A5 gives rise to benign tumors and clone II4 to malignant tumors.

(asRNAs) have been reported to fulfill important functions in gene regulation (for review, see Faghihi and Wahlestedt 2009). Given that the majority of sense transcripts in the eukaryotic genome have counterparts on the opposite strand, we examined whether rRNA genes are also transcribed in antisense orientation. We performed strand-specific RT-PCR, using primers that cover distinct regions of the murine rDNA repeat. Specificity was ensured by reverse transcription with primers linked to the T7 promoter sequence, followed by PCR amplification of the cDNA with an rDNA-specific and the T7 promoter primer. This strand-specific rDNA "walking" revealed low but significant levels of antisense transcripts that cover sequences of the intergenic spacer and the pre-rRNA coding region, but not sequences beyond the downstream transcription terminators $\mathrm{T}_{1}-\mathrm{T}_{10}$ (Fig. 4A). RT-qPCR analysis demonstrated that asRNA is $\sim 30$-fold less abundant than pRNA (data not shown). A heterogeneous population of 12-16-kb RNA molecules was detected on Northern blots using an rDNA promoter probe in sense orientation, indicating that antisense RNA represents long, contiguous transcripts (data not shown). Attempts to map the 5'-end by RACE failed, suggesting that asRNA does not originate from a defined promoter but, rather, from multiple sites within the rDNA arrays.

The level of asRNA varies among different cell lines, in most cases, inversely correlating with the level of $45 \mathrm{~S}$ pre-rRNA. For example, the amount of asRNA was fivefold to sixfold higher in fibroblasts from Werner syndrome (WS) patients than in control fibroblasts, although the amount of pre-rRNA in both cell lines was comparable (Fig. 4B). Conversely, antisense transcripts were found to be down-regulated in transformed cells that exhibit high pre-rRNA synthetic activity. In the experiment shown in
Figure 4C, we compared the relative amount of pre-rRNA and asRNA in HaCaT cells, an immortalized human keratinocyte cell line, with two HaCaT derivatives, A5 and II4, that show varying degrees of tumorigenicity in mice (Boukamp et al. 1988, 1990). Compared to nontumorigenic $\mathrm{HaCaT}$ cells, pre-rRNA synthesis was elevated in the tumor-inducing II4 cell line. The level of asRNA, however, was strongly decreased in both benign A5 and malignant II4 cells, indicating that pre-rRNA and asRNA are regulated differently.

To examine which class of nuclear DNA-dependent RNA polymerases transcribes rDNA in antisense orientation, we treated cells with drugs that specifically inhibit Pol I or Pol II. Treatment with CX-5461, a compound that inhibits Pol I transcription by preventing binding of the transcription initiation factor SL1/TIF-IB to the rDNA promoter (Drygin et al. 2010), did not affect the level of antisense transcripts, suggesting that asRNA is not synthesized by Pol I (Fig. 5A). In support of antisense transcripts being synthesized by Pol II, treatment of HeLa cells with $\alpha$-amanitin, which does not impair Pol I-driven pre-rRNA synthesis, reduced asRNA to a similar degree as c-fos mRNA (Fig. 5B). Significantly, $\alpha$-amanitin did not decrease the synthesis of asRNA in HeLa cells expressing an $\alpha$-amanitin-resistant Pol II mutant (Pol II LS*) (Chapman et al. 2005), reinforcing the notion that antisense transcripts are synthesized by Pol II (Fig. 5B). Finally, we used TIF-IA ${ }^{\mathrm{fl} / \mathrm{fl}}$ mouse embryonic fibroblasts (MEFs) in which the Cre-recombinase can be induced by 4-hydroxytamoxifen (creER ${ }^{\mathrm{T} 2}$ ) (Bierhoff et al. 2008). After induction of Cre-recombinase, TIF-IA was depleted and pre-rRNA synthesis decreased (Fig. 5C). The level of asRNA remained unaffected, reinforcing the fact that Pol II transcribes a fraction of rDNA in antisense orientation. 
A

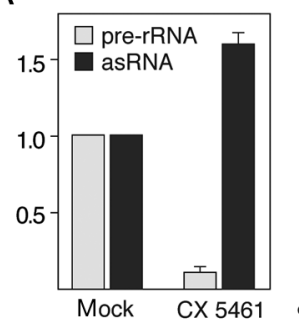

B

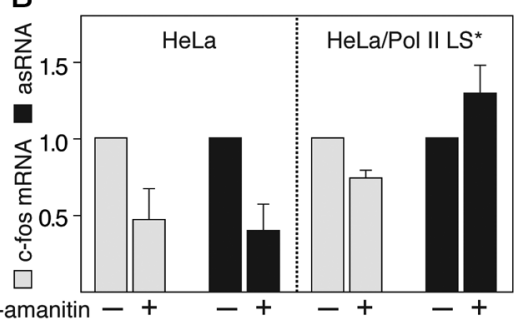

C

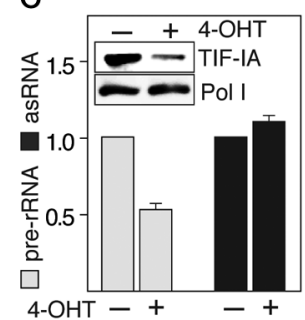

Figure 5. Antisense transcripts are synthesized by RNA Pol II. (A) Inhibition of Pol I does not affect the level of asRNA. NIH3T3 cells were treated with Pol I-specific inhibitor CX-5461 $(10 \mu \mathrm{M}, 2 \mathrm{~h})$. asRNA and pre-rRNA were monitored by RT-qPCR and normalized to levels in mock-treated cells. (B) Inhibition of Pol II impairs synthesis of asRNA. (Left) HeLa cells were mock-treated or treated with $5 \mu \mathrm{g} / \mathrm{mL} \alpha$-amanitin for $12 \mathrm{~h}$, and c-fos mRNA and asRNA were determined by RT-qPCR. (Right) Same experimental approach was used to monitor asRNA in HeLa cells expressing an $\alpha$-amanitin-resistant Pol II mutant (HeLa/Pol II LS*). (C) Depletion of TIF-IA down-regulates pre-rRNA synthesis without affecting asRNA abundance. TIF-IA ${ }^{\mathrm{fl} / \mathrm{fl}}$ MEFs stably expressing creER $^{\mathrm{T} 2}$ were left uninduced or induced with $500 \mathrm{~nm}$ 4-hydroxytamoxifen (4-OHT) for $4 \mathrm{~d}$. TIF-IA levels were monitored on Western blots (inset), and levels of pre-rRNA and asRNA were monitored by RT-qPCR.

\section{ANTISENSE RNA IS DEGRADED BY THE EXOSOME}

The finding that antisense transcripts are underrepresented in steady-state cellular RNA suggests that they are either synthesized with low efficiency or are rapidly degraded. Knowing that the exosome, a complex of $3^{\prime}-5^{\prime}$ exoribonucleases and RNA-binding proteins, has an important role in maturation and degradation of RNAs, we reasoned that exosomal activity may destabilize nucleolar antisense transcripts. Indeed, mRrp6, a catalytic subunit of the murine exosome, colocalizes with UBF in nucleoli (Fig. 6A). Depletion of mRrp6 by RNA interference led to a twofold increase of the level of asRNA (Fig. 6B), supporting the notion that the exosome is involved in the surveillance control of nucleolar asRNA. Knockdown of mTrf4-1 and mTrf4-2, subunits of the TRAMP complex did not affect the level of asRNA, indicating that polyadenylation by $\mathrm{mTrf} 4 \mathrm{~s}$ is not required for exosome-mediated degradation (data not shown). Notably, accumulation of antisense transcripts in mRrp6-depleted cells led to increased occupancy of H4K20me3 and to hypermethylation of rDNA, suggesting that antisense transcripts have a role in establishing heterochromatic features at the rDNA promoter (Fig. 6C).

\section{ANTISENSE TRANSCRIPTS DOWN-REGULATE POL I TRANSCRIPTION}

To get insight into the function of antisense transcripts, we artificially raised the level of antisense transcripts in NIH3T3 cells by transfection of pE3SPmrDNA-43/-142, a Pol I-driven plasmid that directs the synthesis of antisense transcripts comprising murine rDNA sequences from -43 to -142 (Fig. 7A). The level of asRNA increased $\sim 20$-fold compared to cells transfected with the control plasmid pE3SP-MCS (Fig. 7B). Elevated levels of asRNA led to reduced pre-rRNA levels (Fig. 7B), decreased Pol I occupancy, and to an increase of the heterochromatic histone mark H4K20me3 at the rDNA promoter (Fig. 7C). None of the other histone modifications, such as H3K4me3, $\mathrm{H} 3 \mathrm{~K} 9 \mathrm{me} 3$, or H3K27me3, were altered by ectopic asRNA (data not shown). The preferential increase in H4K20me3 suggests that asRNA may recruit the histone methyltransferase Suv4-20h1 and/or Suv4-20h2 to rDNA. Indeed, in vitro binding assays using immobilized GFP-tagged Suv420h1 and Suv4-20h2 revealed that both enzymes bind RNA, exhibiting a strong preference for asRNA as compared to nonspecific RNA or pRNA (Fig. 7E). Together, these results suggest that asRNA contributes to epigenetic regulation of
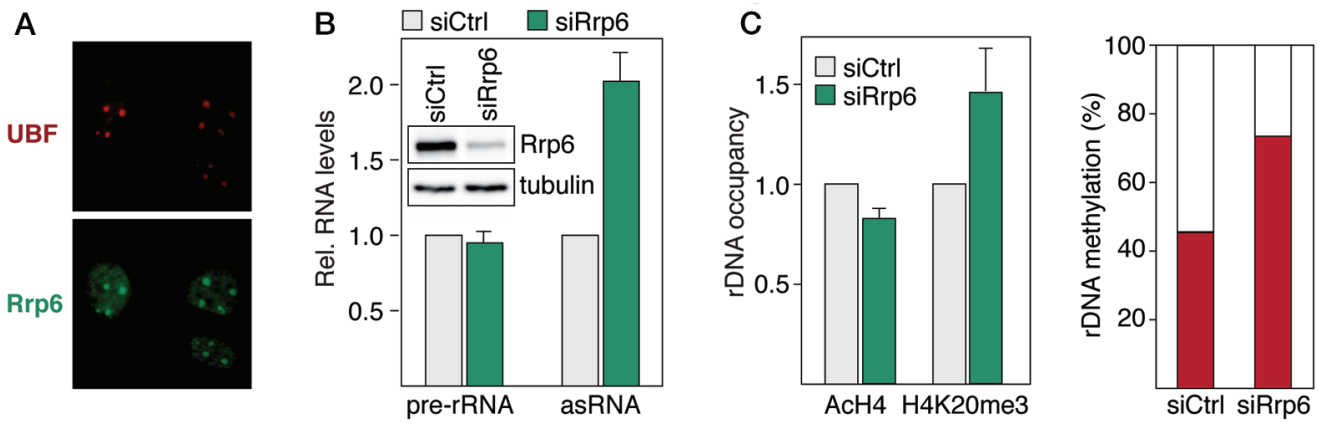

Figure 6. asRNA is degraded by the exosome complex. $(A)$ Indirect fluorescent images showing nucleolar localization of mRrp6 and UBF in NIH3T3 cells. $(B)$ Depletion of Rrp6 leads to increased asRNA levels. NIH3T3 cells were transfected with control siRNA (siCtrl) or siRNAs against Rrp6 (siRrp6), and pre-rRNA and asRNA were monitored by RT-qPCR. (Inset) Western blot demonstrating depletion of mRrp6. (C) Depletion of mRrp6 leads to heterochromatin formation and DNA methylation at the rDNA promoter. (Left) ChIP from NIH3T3 cells transfected with control siRNA or siRrp6 using antibodies against acetylated histone H4 (AcH4) or H4K20me3. (Bars) rDNA occupancy of histone marks normalized to histone H3. (Right) Analysis of DNA methylation by HpaII digestion and qPCR in cells transfected with control siRNA or siRrp6. Shown is the ratio of methylated (red) to unmethylated (white) rDNA. 
A

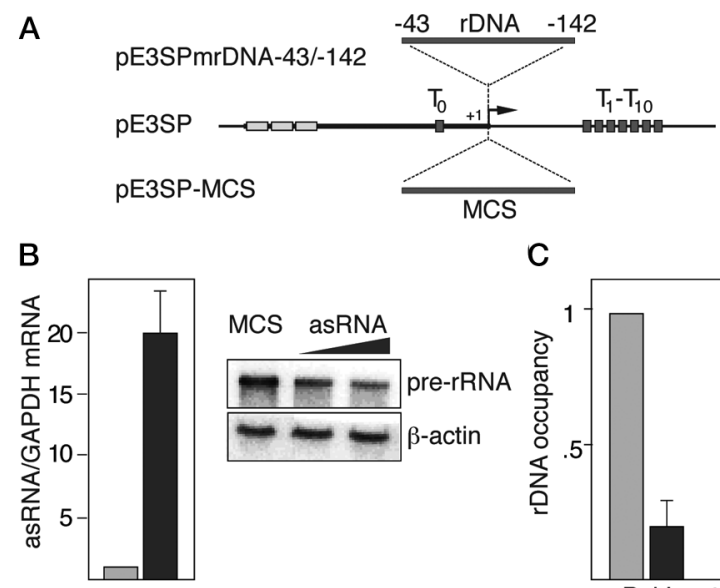

C

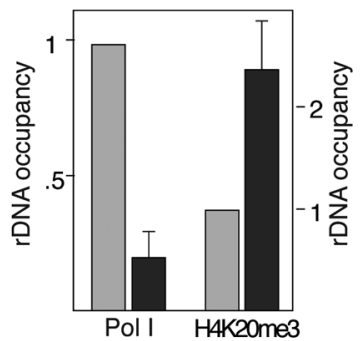

D

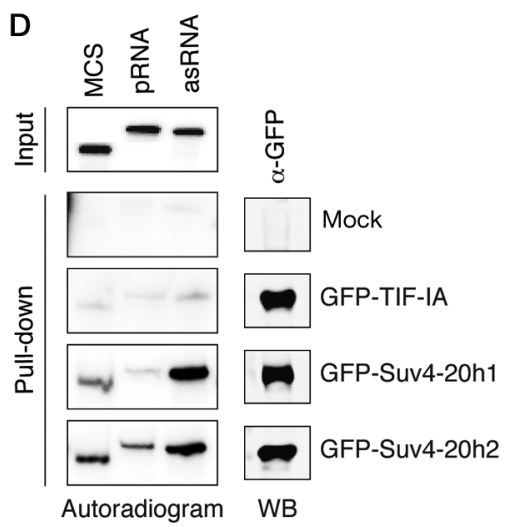

Figure 7. Ectopic asRNA represses rDNA transcription. (A) Pol I-driven plasmid used for the synthesis of ectopic asRNA. pE3SP contains $5^{\prime}$-terminal rDNA sequences (from -639 to -1) including three enhancer elements (light boxes) and upstream terminator element $\mathrm{T}_{0}$ (dark box), fused to $3^{\prime}$-terminal Pol I transcription terminators ( $\mathrm{T}_{1-10}$, dark boxes). pE3SP-mrDNA-43/-142 contains a murine rDNA insert comprising sequences from -43 to -142 in antisense orientation (Brenz-Verca et al. 2007), and pE3SP-MCS contains sequences from the multiple cloning site of pBluescript $\mathrm{SK}^{+} .(B)$ Ectopic asRNA represses pre-rRNA synthesis. (Left) NIH3T3 cells were transfected with pE3SP-MCS (light bar) or pE3SP-mrDNA-43/-142 (dark bar), and expression of asRNA was monitored by RTqPCR. asRNA levels were normalized to GAPDH mRNA levels. (Right) Northern blot showing pre-rRNA and $\beta$-actin mRNA levels in cells transfected with pE3SP-MCS (MCS) or increasing amounts of pE3SP-mrDNA-43/-142 (asRNA). (C) asRNA reduces Pol I occupancy at the rDNA promoter and facilitates heterochromatin formation. Shown is rDNA occupancy of Pol I and H4K20me3 in NIH3T3 cells transfected with pE3SP-MCS (light bars) or pE3SP-mrDNA-43/-142 (dark bars). $(D)$ asRNA binds to Suv4-20h1/2 in vitro. Radiolabeled control RNA (MCS-RNA), pRNA (-205/-1), and asRNA (-1/-205) were incubated with GFP-tagged, immobilized TIF-IA, Suv4-20h1, and Suv4-20h2. After washing, bead-bound RNA was eluted and visualized by autoradiography (left panels). Amount of immobilized proteins was analyzed on Western blots (WB).

pre-rRNA synthesis by recruiting Suv4-20 to rDNA, which, in turn, establishes heterochromatic features at rDNA.

\section{CONCLUDING REMARKS}

With the advent of high-throughput transcriptome analysis techniques, it has become evident that eukaryotic genomes are pervasively transcribed, giving rise to numerous intronic, intergenic, and antisense RNAs lacking protein-coding function. ncRNAs are key players in a variety of epigenetic processes, such as X-chromosome inactivation (Avner and Heard 2001), Hox gene regulation (Koziol and Rinn 2010), and gene imprinting (Nagano and Fraser 2009). Moreover, long ncRNAs mediate epigenetic silencing of tumor-suppressor genes, such as the INK4b/ARF/INK4a locus (Yu et al. 2008; Yap et al. 2010). Our finding that ncRNAs regulate the epigenetic state of rDNA is consistent with recent studies showing that a large fraction of ncRNAs is associated with mammalian chromatin-modifying proteins and may guide them to their target genes (Khalil et al. 2009). Given the close collaboration between NoRC and pRNA, it is noteworthy that pRNA mediates methylation of the rDNA promoter independently from NoRC by directly interacting with $\mathrm{T}_{0}$, a binding site of the transcription factor TTF-I, forming a DNA:RNA triple-helical structure that is recognized by DNMT3b (Schmitz et al. 2010). These observations raise the exciting possibility that triplex-dependent recruitment of DNMT3b to specific gene sequences might be a generally used pathway in epigenetic regulation.

The idea that ncRNA would be well adapted for genespecific transcriptional regulation is not new. Almost 50 years ago, François Jacob and Jacques Monod reasoned that base complementarity would allow RNA to interact specifically with other nucleic-acid sequences (Jacob and Monod 1961). They proposed that the function of some genes is to produce regulatory RNAs that interact by basepairing with specific sequences, either at the transcriptional or posttranscriptional level. With the discovery of RNA interference and its numerous mechanisms in translational repression and heterochromatin formation as well as with the elucidation of the multiple functions of long ncRNAs in gene regulation, this anticipation has proven to be the case. Intriguingly, rRNA genes have proven to represent an ideal system to decipher the mechanism underlying ncRNA-mediated transcriptional regulation. The finding that DNA:RNA triplexes are specifically recognized by DNMT3b suggests that triplex formation between ncRNA and regulatory gene sequences may be a general mechanism that cells use to recruit DNMTs to regions to be methylated. Likewise, asRNA-mediated induction of H4K20 trimethylation by recruitment of Suv4-20h1 and/or Suv4-20h2 might not be restricted to rRNA genes. We envisage that - similar to pRNA-dependent recruitment of NoRC - antisense transcripts may target Suv4-20 enzymes, and possibly other chromatin modifiers to rDNA, thereby establishing a repressive chromatin state. Elucidating this mechanism will provide insights into the function of ncRNAs in the regulation of chromatin-based processes.

\section{ACKNOWLEDGMENTS}

We thank P. Boucamp for HaCat cells, D. Eick for HeLa/ LS* cells, S. Iben for fibroblasts from Werner syndrome patients, and G. Schotta for expression plasmids 
encoding GFP-Suv4-20h1/2. Work in our laboratory has been supported by the Deutsche Forschungsgemeinschaft, the European Community, and the Fonds der Chemischen Industrie.

\section{REFERENCES}

Avner P, Heard E. 2001. X-chromosome inactivation: Counting, choice and initiation. Nat Rev Genet 2: 59-67.

Belotserkovskii BP, De Silva E, Tornaletti S, Wang G, Vasquez KM, Hanawalt PC. 2007. A triplex-forming sequence from the human c-MYC promoter interferes with DNA transcription. $J$ Biol Chem 282: 32433-32441.

Bierhoff H, Dundr M, Michels AA, Grummt I. 2008. Phosphorylation by casein kinase 2 facilitates rRNA gene transcription by promoting dissociation of TIF-IA from elongating RNA polymerase I. Mol Cell Biol 28: 4988-4998.

Bogdanović O, Veenstra GJ. 2009. DNA methylation and methylCpG binding proteins: Developmental requirements and function. Chromosoma 118: 549-565.

Boukamp P, Petrussevska RT, Breitkreutz D, Hornung J, Markham A, Fusenig NE. 1988. Normal keratinization in a spontaneously immortalized aneuploid human keratinocyte cell line. J Cell Biol 106: 761-771.

Boukamp P, Stanbridge EJ, Foo DY, Cerutti PA, Fusenig NE. 1990. c-Ha-ras oncogene expression in immortalized human keratinocytes (HaCaT) alters growth potential in vivo but lacks correlation with malignancy. Cancer Res 50: 2840-2847.

Brenz-Verca MS, Weber P, Mayer C, Graf C, Refojo D, Kühn R, Grummt I, Lutz B. 2007. Development of a species-specific RNA polymerase I-based shRNA expression vector. Nucleic Acids Res 35: e10. doi: 10.1093/nar/gk11045.

Cassidy BG, Yang-Yen HF, Rothblum LI. 1987. Additional RNA polymerase I initiation site within the nontranscribed spacer region of the rat rRNA gene. Mol Cell Biol 7: 2388-2396.

Chapman RD, Conrad M, Eick D. 2005. Role of the mammalian RNA polymerase II C-terminal domain (CTD) nonconsensus repeats in CTD stability and cell proliferation. Mol Cell Biol 17: 7665-7674.

Drygin D, Lin A, Bliesath J, Ho CB, O’Brien SE, Proffitt C, Omori M, Haddach M, Schwaebe MK, Siddiqui-Jain A, et al. 2010. Targeting RNA polymerase I with an oral small molecule CX5461 inhibits ribosomal RNA synthesis and solid tumor growth. Cancer Res 71: 1418-1430.

Evers R, Smid A, Rudloff U, Lottspeich F, Grummt I. 1995. Different domains of the murine RNA polymerase I-specific termination factor mTTF-I serve distinct functions in transcription termination. EMBO J 14: 1248-1256.

Faghihi MA, Wahlestedt C. 2009. Regulatory roles of natural antisense transcripts. Nat Rev Mol Cell Biol 10: 637-643.

Goñi JR, de la Cruz X, Orozco M. 2004. Triplex-forming oligonucleotide target sequences in the human genome. Nucleic Acids Res 32: 354-360.

Hendrich B, Tweedie S. 2003. The methyl-CpG binding domain and the evolving role of DNA methylation in animals. Trends Genet 19: 269-277.

Jacob F, Monod J. 1961. Genetic regulatory mechanisms in the synthesis of proteins. J Mol Biol 3: 318-356.

Khalil AM, Guttman M, Huarte M, Garber M, Raj A, Rivea Morales D, Thomas K, Presser A, Bernstein BE, van Oudenaarden A, et al. 2009. Many human large intergenic noncoding RNAs associate with chromatin-modifying complexes and affect gene expression. Proc Natl Acad Sci 106: 11667-11672.

Kim HG, Miller DM. 1998. A novel-triplex-forming oligonucleotide targeted to human cyclin D1 (bcl-1, proto-oncogene) promoter inhibits transcription in HeLa cells. Biochemistry 37: 2666-2672.

Kobayashi T, Horiuchi T, Tongaonkar P, Vu L, Nomura M. 2004. SIR2 regulates recombination between different rDNA repeats, but not recombination within individual rRNA genes in yeast. Cell 117: 441-453.

Koziol MJ, Rinn JL. 2010. RNA traffic control of chromatin com- plexes. Curr Opin Genet Dev 20: 142-148.

Kuhn A, Grummt I. 1987. A novel promoter in the mouse rDNA spacer is active in vivo and in vitro. EMBO J 6: 3487-3492.

Malecová B, Morris KV. 2010. Transcriptional gene silencing through epigenetic changes mediated by non-coding RNAs. Curr Opin Mol Ther 12: 214-222.

Martianov I, Ramadass A, Serra Barros A, Chow N, Akoulitchev A. 2007. Repression of the human dihydrofolate reductase gene by a non-coding interfering transcript. Nature 445: 666-670.

Mattick JS. 2009. The genetic signatures of noncoding RNAs. PLoS Genet 5: e1000459. doi: 10.1371/journal.pgen.1000459.

Mayer C, Schmitz KM, Li J, Grummt I, Santoro R. 2006. Intergenic transcripts regulate the epigenetic state of rRNA genes. Mol Cell 22: 351-361.

Mayer C, Neubert M, Grummt I. 2008. The structure of NoRCassociated RNA is crucial for targeting the chromatin remodelling complex NoRC to the nucleolus. EMBO Rep 9: 774-780.

Meller VH, Gordadze PR, Park Y, Chu X, Stuckenholz C, Kelley RL, Kuroda MI. 2000. Ordered assembly of roX RNAs into MSL complexes on the dosage-compensated X chromosome in Drosophila. Curr Biol 10: 136-143.

Moss T, Boseley PG, Birnstiel ML. 1980. More ribosomal spacer sequences from Xenopus laevis. Nucleic Acids Res 8: 467-485.

Nagano T, Fraser P. 2009. Emerging similarities in epigenetic gene silencing by long noncoding RNAs. Mamm Genome 20: 557562.

Peng JC, Karpen GH. 2007. H3K9 methylation and RNA interference regulate nucleolar organization and repeated DNA stability. Nat Cell Biol 9: 25-35.

Santoro R, Grummt I. 2001. Molecular mechanisms mediating methylation-dependent silencing of ribosomal gene transcription. Mol Cell 8: 719-725.

Santoro R, Li J, Grummt I. 2002. The nucleolar remodeling complex NoRC mediates heterochromatin formation and silencing of ribosomal gene transcription. Nat Genet 32: 393-396.

Santoro R, Schmitz KM, Sandoval J, Grummt I. 2010. Intergenic transcripts originating from a subclass of ribosomal DNA repeats silence ribosomal RNA genes in trans. EMBO Rep 11: 52-58.

Schmitz KM, Mayer C, Postepska A, Grummt I. 2010. Interaction of noncoding RNA with the rDNA promoter mediates recruitment of DNMT3b and silencing of rRNA genes. Genes Dev 24: 2264-2269.

Sleutels F, Zwart R, Barlow DP. 2002. The non-coding Air RNA is required for silencing autosomal imprinted genes. Nature 415: 810-813.

Strohner R., Nemeth A, Jansa P, Hofmann-Rohrer U, Santoro R, Längst G, Grummt I. 2001. NoRC: A novel member of mammalian ISWI-containing chromatin remodeling machines. EMBO J 20: 4892-4900.

Strohner R, Németh A, Nightingale KP, Grummt I, Becker PB, Längst G. 2004. Recruitment of the nucleolar remodeling complex NoRC establishes ribosomal DNA silencing in chromatin. Mol Cell Biol 24: 1791-1798.

Yap KL, Li S, Muñoz-Cabello AM, Raguz S, Zeng L, Mujtaba S, Gil J, Walsh MJ, Zhou MM. 2010. Molecular interplay of the noncoding RNA ANRIL and methylated histone H3 lysine 27 by polycomb CBX7 in transcriptional silencing of INK $4 \mathrm{a}$. Mol Cell 38: 662-674.

Yu W, Gius D, Onyango P, Muldoon-Jacobs K, Karp J, Feinberg AP, Cui H. 2008. Epigenetic silencing of tumour suppressor gene $\mathrm{p} 15$ by its antisense RNA. Nature 451: 202-206.

Zhou Y, Grummt I. 2005. The PHD finger/bromodomain of NoRC interacts with acetylated histone H4K16 and is sufficient for rDNA silencing. Curr Biol 15: 1434-1438.

Zhou Y, Santoro R, Grummt I. 2002. The chromatin remodeling complex NoRC targets HDAC1 to the ribosomal gene promoter and represses RNA polymerase I transcription. EMBO J 21: 4632-4640.

Zhou Y, Schmitz KM, Mayer C, Yuan X, Akhtar A, Grummt I. 2009. Reversible acetylation of the chromatin remodelling complex NoRC is required for ncRNA dependent transcriptional silencing. Nat Cell Biol 11: 1010-1016. 


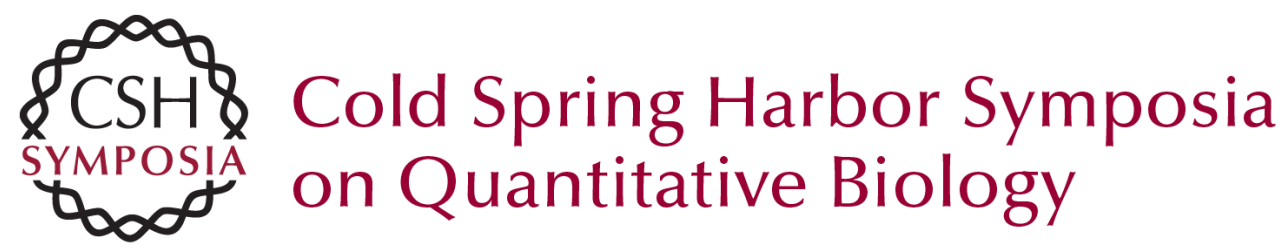

\section{Noncoding Transcripts in Sense and Antisense Orientation Regulate the Epigenetic State of Ribosomal RNA Genes}

H. Bierhoff, K. Schmitz, F. Maass, et al.

Cold Spring Harb Symp Quant Biol 2010 75: 357-364 originally published online April 18, 2011 Access the most recent version at doi:10.1101/sqb.2010.75.060

References This article cites 41 articles, 10 of which can be accessed free at: http://symposium.cshlp.org/content/75/357.full.html\#ref-list-1

License

Email Alerting

Receive free email alerts when new articles cite this article - sign up in Service the box at the top right corner of the article or click here. 